\title{
Preliminary System Design for AMMEQ-1: A Step Towards QKD
}

\author{
By Eugene Kim, ${ }^{1)}$ Xiaofeng Wu, ${ }^{1)}$ Trevor Kwan, ${ }^{1)}$ Xun Sun,${ }^{1)}$ Robert Bedington, ${ }^{2)}$ Xueliang BAI, ${ }^{2)}$ and Alexander LinG ${ }^{2)}$ \\ ${ }^{1)}$ School of Aerospace, Mechanical and Mechatronics Engineering, The University of Sydney, Sydney, Australia \\ ${ }^{2)}$ Centre for Quantum Technologies, National University of Singapore, Singapore, Singapore
}

(Received June 23rd, 2017)

\begin{abstract}
Quantum Key Distribution utilises fundamental quantum properties and mechanics to generate a cryptographic key, ensuring the highest security regardless of advances to computer processing. AMMEQ-1 is a $3 \mathrm{U}$ cubesat being developed by The University of Sydney, Australia hosting a primary payload provided by Centre for Quantum Technologies, Singapore. Its main purpose is to show USYD's capability of developing a satellite using COTS products and to demonstrate various technologies to support future QKD missions. This study describes the mission and overall system design of the cubesat.
\end{abstract}

Key Words: QKD, Cubesat, APD, Lasers, COTS

\section{Introduction}

Secure communication is highly desirable in both military and civilian applications such as banks, corporations and in any situation where sensitive data is transmitted between two parties. With the advancements in quantum computing, traditional encryption deciphering methods which involve the factoring of a very large prime numbers are rendered unsafe and practically useless. In 1977, it was estimated that it would take 40 quadrillion years to decipher the RSA-129.1) However, in 1994, the RSA-129 was deciphered in less than 6 months using a network of computers.

Quantum Key Distribution ${ }^{2)}$ (QKD) addresses the vulnerabilities of traditional methods of secure communication by using quantum properties of light to generate a cryptographic key used to encrypt messages between two parties. Despite advances in computing and the increase in processing power, QKD ensures the highest security in that any external interference to the quantum state will perturb the system and leave a 'footstep' for key-holders to detect.

Ground based QKD systems ${ }^{3)}$ can be implemented using optical fibers for a few hundred kilometers before the losses increase exponentially and becomes infeasible due to a poor signal to noise ratio. Other methods such as free space optical links of varying distances between ground networks ${ }^{4) 5)}$ and airborne systems ${ }^{6) 7)}$ have been investigated. However, these systems require a clear line of sight and losses near the ground caused by atmospheric turbulence and absorption limits transmission to $<200 \mathrm{~km}$ and anything beyond this distance is deemed inefficient.

The implementation of QKD into satellites which orbit above the Earth's atmosphere has many merits as it does not suffer from atmospheric or fiber losses and is able to overcome the distance limitation in the systems mentioned above. Figure 1 shows the various methods of space based QKD involving satellites. Fig. 1a shows a ground to space link where the quantum receiver is placed on a satellite in Low Earth Orbit (LEO) and the photon source is at a ground station. The Canadian Space Agency (CSA) has proposed the Quantum Encryption and Science Satellite ${ }^{8)}$ (QEYSSat) which aims to demonstrate quantum uplink showing which demonstrates this principle. A
Japanese microsatellite SOCRATES ${ }^{9)}$ demonstrates a LEO-toground QKD experiment as shown in Fig.1b. The first QKD satellite launched was Quantum Experiments at Space Scale $(\mathrm{QUESS})^{10)}$ by the Chinese Academy of Science which aims to demonstrate space to ground QKD as shown in Fig. 1c. Figure 1d shows space to space QKD using multiple satellites in orbit. A network of small satellites flying in formation can possibly be utilised to realize this system.

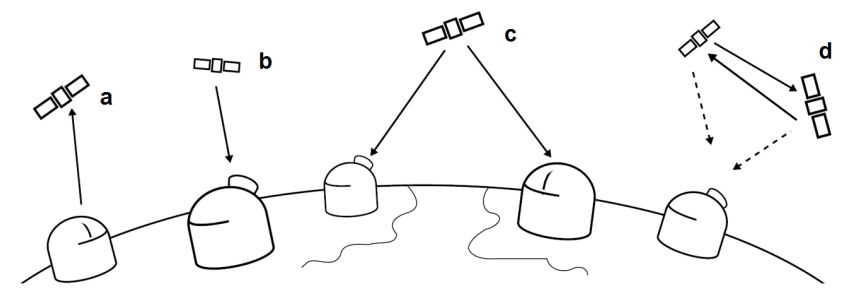

Fig. 1. LEO space based QKD applications. a) Ground to space transmission. b) Space to ground transmission. c) Simultaneous space to ground transmission. d) Space to space QKD.

The School of Aerospace, Mechanical and Mechatronics Engineering (AMME) at The University of Sydney (USYD) is working with Centre for Quantum Technologies (CQT), Singapore to develop a 3U cubesat "AMMEQ-1" which tests various subsystems in support of the CQT SpooQySats program which assesses how different APDs and lasers operate and uphold when exposed to the harsh space environment. It will test the technology required for future space based QKD missions. A cubesat platform is chosen for the easily accessible components and number of launch options available to deliver the payload into space. It will be developed using commercial-off-theshelf (COTS) products to demonstrate the reliability and capability of operating COTS products in space.

USYD has extensive experience in developing flight-ready space projects which have either launched or demonstrated practical functionality to operate in the space environment. USYD has developed the i-Inspire (initial - INtegrated SPectrograph, Imager and Radiation Explorer), ${ }^{11)}$ to demonstrate various technologies and in-house developed instruments to support the next generation satellite, Inspire-2. It demonstrated USYD's capability to design, develop, test and operate a spacecraft. Inspire- $2^{12)}$ is a $2 \mathrm{U}$ cubesat development project led by 
USYD for participation in the QB50 program. ${ }^{13)}$ It is the first Australian cubesat to be launched into space with two other Australian cubesats. Inspire-2 houses various payloads to conduct experiments in the lower thermosphere for approximately 12 months before re-entering the atmosphere.

CQT also has experience in flight-proven space payloads and are working on an iterative in-orbit demonstration programme to develop the space-qualified optoelectronic technologies required to achieve long distance, free-space QKD from CubeSat platforms. So far these have been successfully demonstrated as $1 \mathrm{U}$-sized correlated photon sources on a weather balloon, ${ }^{14)}$ a student-led CubeSat, ${ }^{15)}$ and an exploding rocket. ${ }^{16)}$ Building on these tests, current efforts at CQT are directed towards developing $2 \mathrm{U}$-sized, polarisation entangled photon pair sources. These will be tested on 3U CubeSats including dedicated SpooQySat missions run by CQT. ${ }^{17)}$ Once the component technologies have been fully developed and tested in these programs, future missions can be planned to demonstrate satelliteto-ground, and satellite-to-satellite QKD using free-space optical communication links.

In this study, the mission profile of AMMEQ-1 is described according to the respective payloads, and a success criteria is defined accordingly. The success criteria is based on the successful operation of the payloads and execution of the missions. A detailed description of the primary payload developed by CQT is presented and the secondary payloads including the inhouse developed components are described in detail. The study also presents the overall system components and subsystems of the cubesat.

\section{Payloads and Mission Profile}

The mission profile consists of several missions derived from the primary and secondary payloads. The primary mission re-volves around the payload developed by CQT while the secondary missions are based on the operation of payloads developed in-house at AMME: a gamma ray microdosimeter and reaction wheels.

\subsection{Primary payload: SPEQS}

CQT cubesat payloads are self-contained experiments known collectively as SPEQS (Small Photon Entangling Quantum Systems). Depending on development constraints, the primary payload on AMMEQ-1 will be either a SPEQS unit or a test-bench of candidate subsystems. The testing of APDs and lasers in the space environment is crucial step in any space-based single photon counting experiment. Figure 2 shows an example of a $1 \mathrm{U}$ correlated photon SPEQS device from earlier missions.

These sources produce pairs of photons which are then detected and analysed within the SPEQS payload. These setups do not perform QKD themselves, but are used to verify the inorbit performance of the components and subsystems which are all enclosed within a light-tight cover. The optical assembly sits atop the electronics board ${ }^{18)}$ which provides the necessary voltages and control signals, and processes the signals from the detectors. It is controlled independently from the satellite by a dedicated onboard PSOC 3 controller which can receive commands from the satellite, and send the experiment data packets, through a UART interface. Figure 3 shows the optical compo-
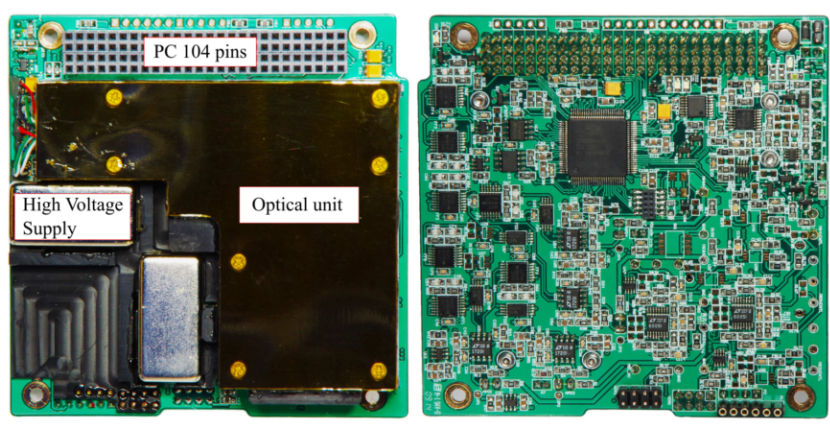

Fig. 2. Top and bottom view of a SPEQS device from earlier missions. Upcoming SPEQS devices will at least double the size and will be independent of the PC104 stack. $^{18)}$

nents that will be needed for a high brightness (QKD-ready) SPEQS device, and indicates the iterative development cycle being pursued.

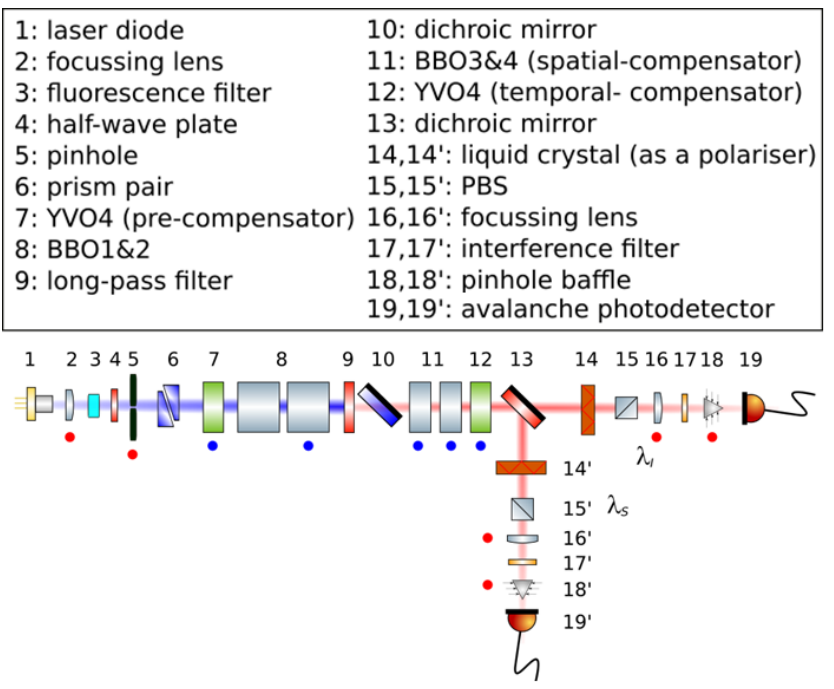

Fig. 3. Optical layouts for SPEQS. Current development omit components marked with red dots. Previously launched devices also omit components marked with blue dots. ${ }^{17), 19)}$

Key components to verify include the laser diode, the flexure stages that support the BBO crystals, and the avalanche photo diodes (APDs). ${ }^{20)}$ Key subsystems to verify are those that control the stability of the APDs, laser and liquid crystal polarisation rotators as the thermal conditions vary and as radiation damage increases. Testing multiple variants of such devices and subsystems in multiple missions provides CQT with valuable experience in a variety of orbital environments and helps define and de-risk future, more complex space-based QKD experiments.

\subsection{Secondary payloads}

AMMEQ-1 also consists of several secondary payloads to support the primary payload whilst having respective missions of its own. In addition to the CQT primary payload,

AMMEQ-1 houses a gamma ray microdosimeter developed in-house at AMME and an optical camera for Earth observation missions. Furthermore, the reaction wheels which are also developed in-house serves the purpose of a technology demonstration.

\subsubsection{SUGAR dosimeter}

The SUGAR ${ }^{21)}$ (Sydney University GAmma Ray) microdosimeter is an in-house developed payload which has already 
flown on previous missions such as i-Inspire and Inspire-2. It will support the primary mission to assess how different APDs and lasers operate in space by measuring the dose of incoming ionised radiation and will observe any changes or faults in the primary payload as a result of exposure to the space environment. It uses very low power $(3.3 \mathrm{~V})$, and takes up very little mass and volume.

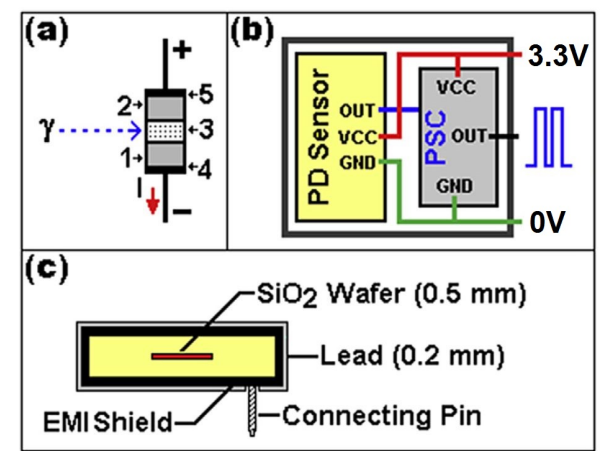

Fig. 4. a) Schematic showing the principle of the PIN diode. b) Schematic diagram of the SUGAR dosimeter showing the PIN diode interfaced to the Pulse Shaping Circuit (PSC). c) Cross section of the PIN diode dosimeter module. $^{21)}$

The signal output is converted into a steady pulse train using a twin NOR gate. A digital temperature sensor attached close to the dosimeter monitors temperature fluctuations and corrects the detector output. The schematic diagram of SUGAR dosimeter principle is depicted in Fig. 4. Extensive environmental testing was conducted on the SUGAR dosimeter: random vibration excitation ranging from 0 to $1000 \mathrm{~Hz}$, thermal testing ranging -20 to $50^{\circ} \mathrm{C}$, and vacuum testing under $10^{-5} \mathrm{mBar}$.

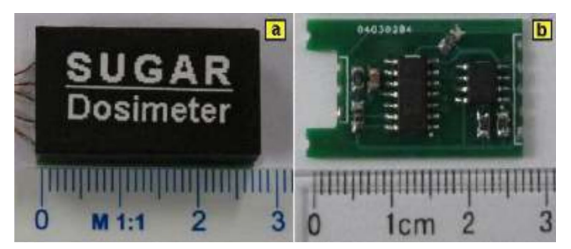

Fig. 5. a) The in-house developed SUGAR (Sydney University GAmma Ray) dosimeter assembled on the PCB. b) The PCB with the components not including the microprocessor and SD card.

The performance and operation of the payload was validated through a high altitude balloon launch (30km altitude) in June 2015 at West Wyalong (3351'S, $\left.147^{\circ} 24^{\prime} \mathrm{E}\right)$, Australia. The full specifications of SUGAR can be seen in Table 1 with additional results from the high altitude balloon launch test. Figure 5 shows the actual flight-ready payload which has been tested and validated.

\subsubsection{Reaction wheels}

The reaction wheels are also developed in-house and serve the purpose of a technology demonstration of using COTS com-ponents to develop an attitude actuator for high pointing accu-racy. AMMEQ-1 does not have any point-to-point communica-tion payloads and no attitude pointing requirements to execute the primary mission. However, the reaction wheels are intended to test technology for future QKD missions which require very high pointing accuracy to establish a successful optical commu-nication link. In order to demonstrate quantum or laser commu-nication, a pointing accuracy of at least 0.01 degree or 36 arc-seconds is required. Although this requirement may be difficult
Table 1. Specifications of SUGAR dosimeter including data collected during high altitude balloon launch. ${ }^{21)}$

\begin{aligned} & \hline Specification Value/Result \\ & \hline Mass including PCB $9.6 \mathrm{~g} \\ &$ Dimensions $28 \times 16 \mathrm{~mm} \\ &$ Operating voltage $3.3 \mathrm{~V} \\ &$ Current drain $2.9 \mathrm{~mA} \\ &$ Power consumption $1.5 \mathrm{~mW} \\ &{ }^{60}$ CO-gamma response 5.8 counts. $\mathrm{min}^{-1} / \mu \mathrm{S} v \mathrm{~h}^{-1} \pm 1.5 \% \\ &$ Temperature sensitivity $200-333 \mathrm{~K} \\ &$ Vibration immunity Intact at $2.8 \mathrm{G} \\ &$ Microwave immunity Guaranteed \\ & \hline\end{aligned}

to be realized with a cubesat platform, developing a reaction wheel with high pointing accuracy which can be used in addition to optical steering is possible.

The reaction wheel design is based on a dual actuator system. There are a few brushless motor based micro reaction wheels available in the market. However, these types of actuators are unable to achieve high pointing accuracy due to the high attitude sensitivity of light weight cubesats and the low speed nonlinearity of brushless motors.

In this reaction wheel design, ultrasonic piezoelectric motors are embedded into an in-house developed EC brushless motor reaction wheel system. Ultrasonic piezoelectric motors are a new type of actuator that is driven by mechanical vibration in the ultrasonic range. The mechanical vibration is excited using piezoelectric ceramics which expand or contract under an electric field. Ultrasonic motors are becoming excellent actuators for precise micromechanical systems and is adopted to optimise the performance of the attitude control system in the low speed range. To achieve three-axis attitude control with redundancy, an appropriate reaction wheel housing is also designed in a tetrahedral configuration (seen in Fig. 6).

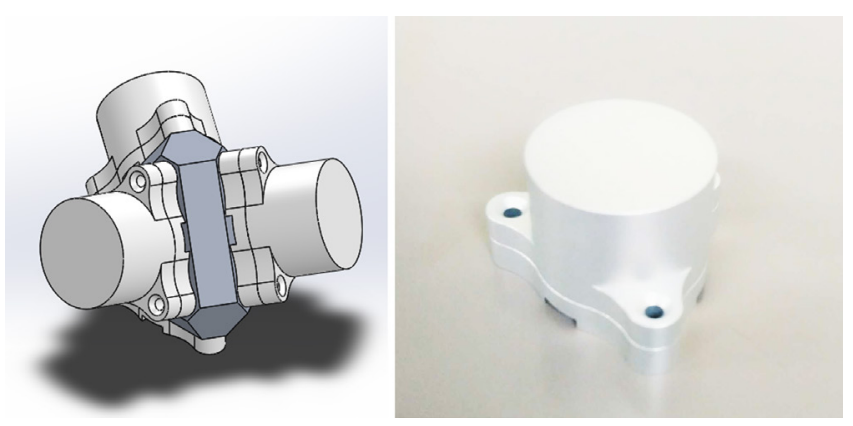

Fig. 6. Left: CAD model of the proposed reaction wheel design in tetrahedral pyramid configuration. Right: Micro reaction wheel casing.

Precise three-axis attitude control is a key element for any mission that requires high pointing accuracy and extends the utility of cubesats with such control. This reaction wheel system will be tested on orbit and used for future QKD missions, and will be validated through the secondary Earth observation mission which uses the optical camera.

\subsubsection{Optical camera for Earth observation}

A secondary science mission is used to validate the pointing accuracy of the reaction wheels developed in-house. The Nanocam $\left.\mathrm{C}_{1} \mathrm{U}^{22}\right) 70 \mathrm{~mm}$ is manufactured by Gomspace and is used for Earth observation and remote sensing in AMMEQ-1. 
The camera has a 3 megapixel color CMOS sensor and has a ground sampling distance of $<30 \mathrm{~m} /$ pixel at $650 \mathrm{~km}$.

As the primary payload operates independently to the attitude of the satellite, the reaction wheels are instead used to control the attitude for Earth observation. Earth observation from space is able to provide valuable information from global environmental and weather changes to disaster monitoring by covering a wide area in a very short time. There are two modes of operation for the optical camera: a single point tracking mode and multi-target continuous shooting mode.

Figure 7 depicts the first mode of the secondary Earth observation mission: single point tracking mode. In this mode, the satellite will track a single location on the ground and take multiple photos as it passes over the target. It aims to take 3 photos: once at -30 degrees to the target, once at nadir and once at +30 degrees to the target. This allows for visual comparison of a particular site and shows any changes occurring in a very short period. Tracking of the target is done using the ADCS and reaction wheels to control the attitude of the satellite as it performs this maneuver. The purpose of this attitude maneuver is also to demonstrate the pointing accuracy of the reaction wheels and ultimately to show that it is capable of high pointing accuracy required to support future QKD missions.

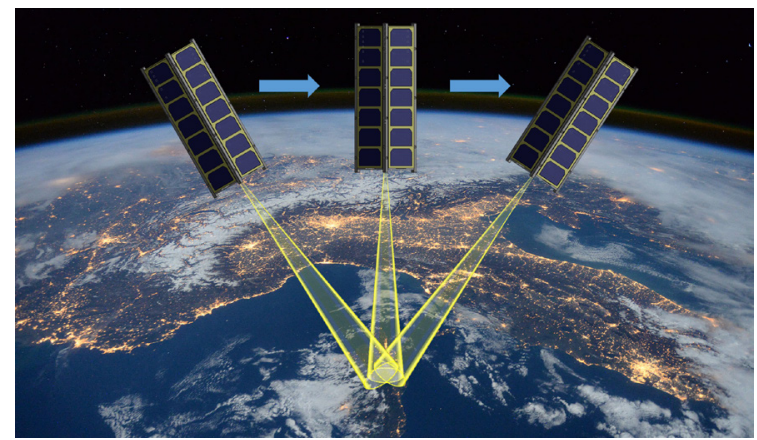

Fig. 7. Single point tracking mode.

The second mode is a multi-target continuous shooting mode where the satellite will shoot various targets of interest and take continuous shots normally without any special attitude maneuver from one target to the next. This shooting mode is depicted in Fig. 8.

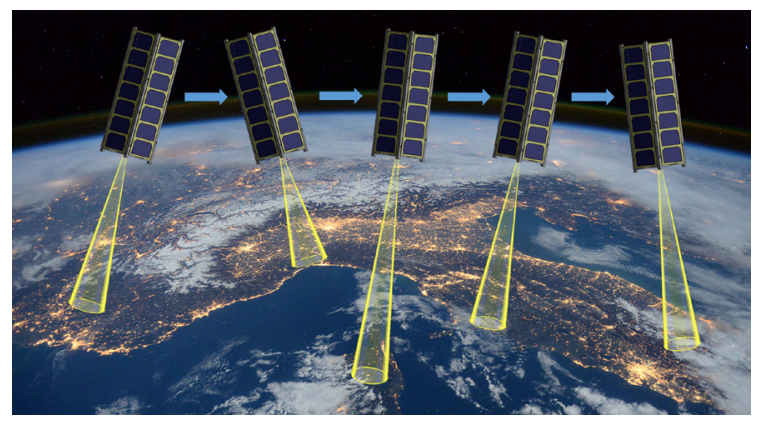

Fig. 8. Multiple point continuous shooting mode.

\subsection{Success criteria}

The success criteria for the mission profile is defined using a point system corresponding to the various missions and payload. The minimum success is defined to be a successful RF connection and downlink of telemetry data upon deployment and start-up of the satellite. Partial success is defined as the successful operation of at least the primary payload and any combination of the secondary payloads. The optical Earth observation mission success is dependent on the successful downlink of photographs from two modes of operation for the payload - 100 points for successfully photographing from either the single point tracking mode or multi-target continuous mode and 200 points for the successful operation of both modes. Full success is defined as the successful execution and operation of all payloads and missions with respective data downlink for each part of the mission. Table 2 shows the list of success criteria and respective points allocated to each criterion.

Table 2. Point system to determine mission success.

\begin{tabular}{lc}
\hline Success Criteria & Points \\
\hline RF and telemetry downlink & 50 \\
Data acquisition from microdosimeter & 100 \\
Attitude control with reaction wheels & 100 \\
Photo capture in one mode & 100 \\
Photo capture in both modes & 200 \\
Operation of primary payload \& data downlink & 300 \\
\hline
\end{tabular}

\section{Orbit Analysis}

The orbit of the satellite is not important as there are no orbital requirements or conditions required to execute the primary mission except for communication purposes. However, due to constraints of the launch vehicle and the orbit of the Cygnus spacecraft used to deliver the satellite, the orbit of the International Space Station (ISS) is currently being considered. The Systems Tool Kit (STK) is used to simulate and analyse the orbit and an altitude of $500 \mathrm{~km}$ (refer to Launch Opportunities) was chosen with an inclination of 51.64 degrees.

The ground segment location was chosen as Sydney and the mean access time (AOS to LOS) per pass was calculated to be 577.9 seconds for an average of 7 passes daily averaged over a week. The orbital path over Australia on a certain day is shown in Figure 9. From this orbit, the average period in sunlight can be calculated for the duty cycle generation used in the electrical power system.

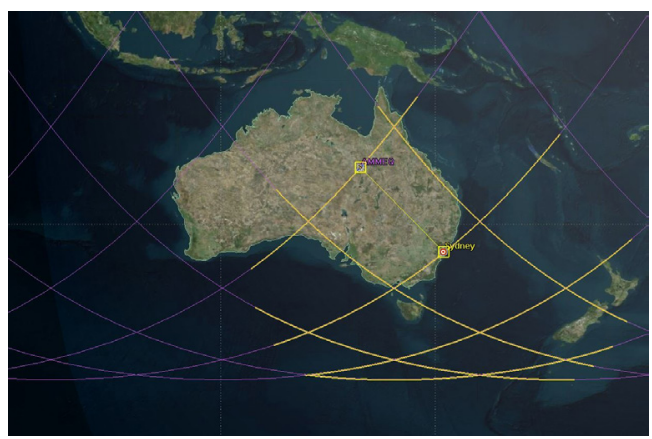

Fig. 9. Orbit path over Australia where the yellow lines indicate the path showing line of sight between the satellite and ground station in Sydney.

\section{Launch Opportunities}

Another reason for the increasing popularity in cubesat development is the ability to send the cubesat to the ISS as cargo via Dragon, ${ }^{23)}$ Cygnus, ${ }^{24)}$ or $\mathrm{HTV}^{25)}$ and then deployed 
from the NanoRacks Cubesat Deployer ${ }^{26)}$ or the Japanese Small Satellite Orbital Deployer. ${ }^{27)}$ Transportation of the cubesat as a cargo means reduced exposure to severe vibrations and loads during launch which may otherwise cause malfunction. This option allows flexibility in that the cubesat can be delivered on any resupply mission to the ISS and does not require specific designation of a launch vehicle.

However, immediate deployment from the ISS is not guaranteed and must adhere to the schedules of the astronauts onboard. Additionally, the orbit of the cubesat is similar to or lower than the ISS orbit which does not guarantee a long lifetime. Recently, NanoRacks has integrated the ability to deploy cubesats from the Cygnus spacecraft after departure from the ISS into a higher $500 \mathrm{~km}$ orbit. ${ }^{28)}$ This is favourable as it will be able to inject the Cubesat into a much higher orbit $(500 \mathrm{~km})$, adding an additional two years before re-entry into the atmosphere. AMME is currently in discussions with NanoRacks to launch and deploy AMMEQ-1 from the external platform on Cygnus directly into orbit. The specific launch date has not yet been determined but the completion of the flight model is targeted to be late 2018 .

\section{Satellite Structure and Subsystems}

The satellite bus is based on the $3 \mathrm{U}$ cubesat standard with dimensions of $30 \times 10 \times 10 \mathrm{~cm}$. A $3 \mathrm{U}$ bus based on the Gomspace satellite bus and was selected to accommodate the primary payload developed by CQT. The satellite component layout was based around the primary payload and to position the radio and antenna in the structure away from the primary payload to avoid interference during the primary mission. Each solar cell covers a surface area of approximately $10 \mathrm{~cm}$ by $5 \mathrm{~cm}$ and thus one panel measuring $30 \mathrm{~cm}$ by $10 \mathrm{~cm}$ can accommodate 6 cells. Each panel is mounted onto exterior sides of the satellite structure.

\subsection{Attitude determination and control system}

The Attitude Determination and Control System (ADCS) does not play a crucial role in executing the primary mission as the primary payload does not require attitude pointing. However, other operation modes require use of the ADCS to a certain extent. When the satellite is in the detumble, communication or secondary science mission mode, it will use the ADCS to make attitude maneuvers and corrections.

Attitude actuators consist of a 3 axis magnetorquer as the primary actuator and a set of four reaction wheels which will both be developed in-house at the University. The mangetorquers are used to detumble the satellite after injection into orbit and during normal and communication modes as required. The reaction wheels are used to demonstrate high accuracy pointing technology developed in-house and to control the attitude during the secondary science mission. As seen in Fig. 7, the satellite is required to maintain the pointing at a single target at is flies over to capture photos of the same target from various angles.

Attitude sensors consist of five sun sensors from Hamamatsu and an Inertial Measurement Unit (IMU) from Analog Devices. The IMU, ADIS16405 (Fig. 10a), is an inertial system measuring $23 \times 23 \times 23 \mathrm{~mm}$ and consists of: a triaxial gyroscope with digital range scaling $\pm 75^{\circ} / \mathrm{sec}, \pm 150^{\circ} / \mathrm{sec}, \pm 300^{\circ} / \mathrm{sec}$, triaxial accelerometer $( \pm 18 g)$ and a triaxial magnetometer ( \pm 2.5 gauss).
Orbit determination and positioning is carried out by a OEMV-1 Single-Frequency GPS Receiver by NovAtel (Fig. 10b). It has a Single Point L1 accuracy of $1.5 \mathrm{~m}$ and can be accurate up to $0.2 \mathrm{~m}$ using RT-20 corrections.
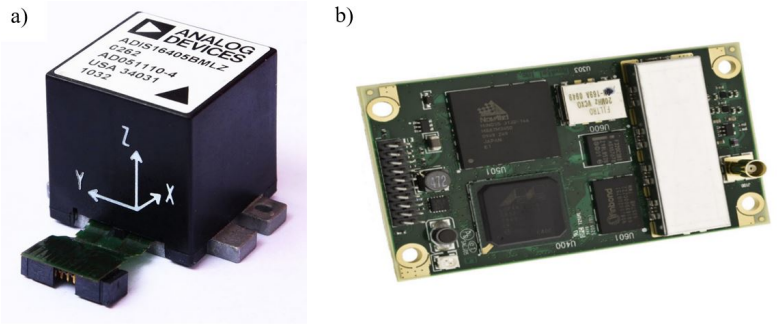

Fig. 10. a) IMU ADIS16405 from Analog Devices. b) OEMV-1 SingleFrequency GPS Receiver from NovAtel. Both instruments partially make up the ADCS.

\subsection{On board computer}

To accommodate the power requirements of the payloads and different modes of operation, the On-Board Computer (OBC) is responsible for the Command and Data Handling ( $\mathrm{CDH})$ of the satellite. The $\mathrm{OBC}$ is designed to maintain the satellite in a stable mode until enough energy is stored in the batteries to carry out the missions. The CubeSat Kit ${ }^{\mathrm{TM}}$ Pluggable Processor Module (PPM) B1 Pumpkin board is responsible for the $\mathrm{CDH}$ and telemetry tracking and command.

\subsection{Communications subsystem}

AMMEQ-1 will utilise amateur radio frequency in the VHF and UHF bands for all communication uplink and downlink transmission. It attempts to demonstrate the reliability of using readily available COTS products. An advantage of using the amateur radio frequency is that anyone who possess an amateur radio license will be able to receive telemetry data from AMMEQ-1 and report it from anywhere in the world. The communications subsystems consists of an ISIS VHF/UHF TRXUV Transceiver (Fig. 11) and ISIS UHF/VHF Antenna System with dual dipole configuration. The transceiver uses BPSK downlink modulation and has telemetry, telecommand and beacon capability on a single board. The ground station at AMME and CQT will be used for command uplink and communication with the satellite from the ground.

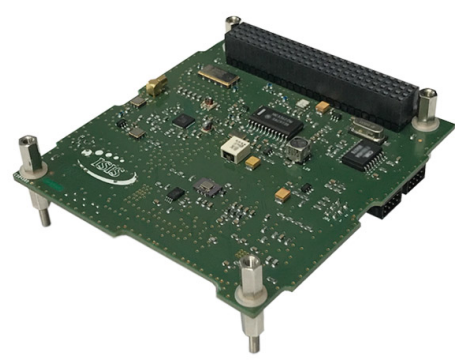

Fig. 11. ISIS VHF/UHF TRXUV Transceiver to be used in conjunction with the ISIS Antenna System.

\subsection{Electrical power system}

Currently, there are several variants of the SPEQS series of instruments being developed at CQT which are being considered for the primary mission. There is a $2.5 \mathrm{~W}$ variant which has a maximum daily energy consumption of $12 \mathrm{Wh}$ (for $10 \%$ duty cycle, average daily duration of operation) and a $10 \mathrm{~W}$ 
variant which has a maximum daily consumption of $24 \mathrm{Wh}$ (for $10 \%$ duty cycle). However, future payloads may have a higher power requirement if active detector cooling systems are used and thus this study will consider the worst case scenario of a payload requiring $20 \mathrm{~W}$ with $10 \%$ duty cycle (average duration of operation daily) and maximum daily energy consumption of 48Wh. These possibilities are listed in Table 3 with the respective power consumption of each payload. Although not required, this study will base the EPS design on a payload requiring $20 \mathrm{~W}$ at $10 \%$ duty cycle in anticipation of and to test technologies for future payloads required for QKD. This case also involves the highest power consumption and hence requires the comparatively higher rated components.

\begin{tabular}{ccc} 
Table 3. & Power requirement for the various SPEQS series instruments. \\
\hline $\begin{array}{c}\text { Power } \\
\text { Requirement (W) }\end{array}$ & $\begin{array}{c}\text { Duty Cycle } \\
\text { (\% of day) }\end{array}$ & $\begin{array}{c}\text { Daily Energy } \\
\text { Consumption (Wh) }\end{array}$ \\
\hline 2.5 & 20 & 12 \\
10 & 10 & 24 \\
20 & 10 & 48 \\
\hline
\end{tabular}

A payload requiring $20 \mathrm{~W}$ of power integrated into a $3 \mathrm{U}$ cubesat is indeed a very high power density component and implies the requirement of a high density EPS design. Thus, another motivation of this study is the design of a high power density EPS that is tailored to support any payload of up to $20 \mathrm{~W}$ and specifically a future SPEQS payload which may require $20 \mathrm{~W}$ to operate. Figure 12 shows the overall architecture of the proposed EPS design where each nodal point of the power circuit should be able to withstand a voltage of up to $20 \mathrm{~V}$. The EPS design should also allow for a maximum continuous current of $5 \mathrm{~A}$ at every power line of the circuit without any component damage. In doing so, each DC-DC converter should be able to handle a maximum power of $100 \mathrm{~W}$ which meets the worst case scenario payload requirement.

\begin{tabular}{|c|c|c|c|c|}
\hline PV Panel 1 & Non-Inverting & V_Batt & Buck 1 & 5V (Payload) \\
\hline & Duch-Doust & & Buck 2 & 5V (Other) \\
\hline PV Panel 2 & Non-Inverting & \multirow{2}{*}{ Batteries } & Buck 3 & $3.3 \mathrm{~V}$ \\
\hline & DUCK-DOUSt & & & ge \\
\hline
\end{tabular}

Fig. 12. Proposed design of the EPS architecture for AMMEQ-1.

It is proposed that the converters of the EPS architecture be locally controlled via a MSP430F5529 digital implementation. To maximise the power extracted from the solar panels, the input converters will be controlled via a Maximum Power Point Tracking (MPPT) algorithm. These converters are chosen as the Non-Inverting Buck-Boost (NIBB) converters specifically as this converter allows for a universal range of voltage gains. This is important as the solar panel voltage is often stochastic in nature. The load converters are then controlled via digitally implemented PI controllers. A more in-depth description of the AMMEQ-1 EPS system and simulation results can be found in this study. ${ }^{31)}$

Power for the satellite will be supplied by the solar array panels which consists of Azur Space Solar Power GmbH - TJ Solar Cell Assembly 3G30A ${ }^{29)}$ shown in Fig. 13a. The cubesat consists of 4 solar panels comprised of 6 Azur Space solar cells (Fig. 13a).
The batteries to be used as part of the EPS is from Gomspace and consists of eight 3.7V lithium ion batteries ${ }^{30)}$ of 18650 form factor connected in the four series in two parallel configurations. It will provide up to 77Wh of power and the battery setup can be seen in Fig. 13b. It is crucial that the battery provides energy to the satellite whilst in the eclipse and thus the minimum battery capacity is estimated based on the average energy consumption during this stage. ${ }^{31)}$ a)

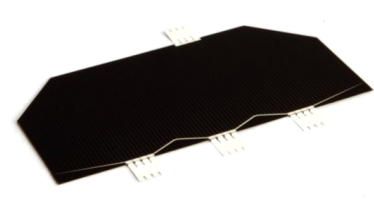

b)

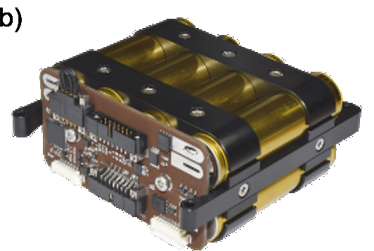

Fig. 13. a) Triple Junction GaAs Solar Cell Assembly from Azur Space to be used in AMMEQ-1. b) GomSpace batteries which will provide 77Wh of energy when 8 are installed.

\section{Conclusion and Future Works}

This paper has presented the proposal and preliminary design of a $3 \mathrm{U}$ cubesat "AMMEQ-1" which is to be developed and launched as a pre-cursor to more sophisticated cubesats with QKD missions. AMMEQ-1 is being developed at The University of Sydney in support of the SpooQySats program at CQT. It is a $3 \mathrm{U}$ cubesat with a primary mission of assessing the operation of APDs and lasers supporting the SPEQS instruments in the space environment. It also houses several secondary payloads: the SUGAR microdosimeter and reaction wheels which demonstrate technology developed in-house and also support the primary mission. An optical camera is also installed for a secondary Earth observation mission. This project demonstrates the capability of using COTS products in a space environment as part of a mission to test technologies for more complex payloads for the future.

\section{Acknowledgments}

This work is partially supported by the National Research Foundation, Prime Minister's Office, Singapore (Award No. NRF-CRP12-2013-02). The Author would also like to thank Yijun Huang. Thank you to Nina and Sara for their support and guidance throughout this project.

\section{References}

1) Gardner, M. Mathematical Games A new kind of cipher that would take millions of years to break. Scientific American Inc. 1977. pp. 120-124.

2) Bennett, C. H., and Brassard, G: Quantum cryptography: public key distribution and coin tossing, Proceedings of the IEEE International Conference on Computers, Systems and Signal Processing, (1984), pp. 175-179

3) Stucki, D., Walenta, N., Vannel, F., Thew, R. T., Gisin, N., Zbinden, H., Gray, S., Towery, C. R., and Ten, S.: High rate, long-distance quantum key distribution over $250 \mathrm{~km}$ of ultra low loss fibres. New Journal of Physics, 11(7) (2009), pp. 9. 
4) Ursin, R., Tiefenbacher, F.,Schmitt-Manderbach, T., Weier, H., et al: Entanglement-based quantum communication over $144 \mathrm{~km}$, Nature Physics, 3 (2007), pp. 481-486.

5) Schmitt-Manderbach, T., Weier, H., Furst, M., Ursin, R., et al.: Experimental demonstration of free-Space decoy-state quantum key distribution over 144 km, Physical Review Letters, 98 (2007), pp. 9812190.

6) Nauerth, S., Moll, F., Rau, M., Fuchs, C., Horwath, J., Frick, S., and Weinfurter, H.: Air-to-ground quantum communication, Nature Photonics, 7 (2013), pp. 382-286.

7) Pugh, C., Kaiser, S., Bourgoin, J. P., Jin, J., Sultana, N., Agne, S., et al.: Airborne demonstration of a quantum key distribution receiver payload, arXiv preprint arXiv:1612.06396, 2016.

8) D'Souza, I., Hudson, D., Evans, C., Choi, E., Jennewein, T., and Sarda, K.,: The QEYSSat mission: demonstrating global quantum key distribution using a microsatellite, Proceedings of the 26th Annual AIAA/USU Conference on Small Satellites, 2012.

9) Takenaka, H., Carrasco-Casado, A., Fujiwara, M., Kitamura, M., Sasaki, M., \& Toyoshima, M.: Satellite-to-ground quantum communication using a 50-kg-class micro-satellite, Nature Photonics, 11 (2017), pp. 502508. DOI: 10.1038/NPHOTON.2017.107

10) Yin, J., Cao, Y., Li, Y. H., Liao, S. K., Zhang, L., Ren, J. G., et al.: Satellite-based entanglement distribution over 1200 kilometers, Science, 356(6343) (2017), pp. 1140-1144.

11) Fogarty, L., Cairns, I., Bland-Hawthorn, J., Wu, X., Betters, C., Funamoto, J. et al.: The initial - INtegrated SPectrograph, Imager and Radiation Explorer (i-INSPIRE) - a university satellite project. Proceedings of the 11th Australian Space Science Conference, 2011.

12) The University of Sydney - Inspire 2 Cubesat, http://sydney.edu.au/inspire-cubesat/ (accessed March 24, 2017)

13) Von Karman Institute - QB50, https://www.qb50.eu/ (accessed March 24, 2017)

14) Zhongkan, T., Chandrasekara, R., Sean, Y. Y., Cheng, C., Wildfeuer, C. and Ling, A.: Near-space flight of a correlated photon system, Sci. Rep., 4 (2014), pp. 6366.

15) Tang, Z., Chandrasekara, R., Tan, Y. C., Cheng, C., Sha, L., Hiang, G. C., Oi, D. K. and Ling, A.: Generation and analysis of correlated pairs of photons aboard a nanosatellite, Physical Review Applied, 5(5) (2016), pp. 054022.

16) Tang, Z., Chandrasekara, R., Tan, Y.C., Cheng, C., Durak, K. and Ling, A.: The photon pair source that survived a rocket explosion. Scientific Reports, 6 (2016).

17) Bedington, R., Bai, X., Truong-Cao, E., Tan, Y. C., Durak, K., Zafra, A. V., Grieve, J. A., Oi, D. K. and Ling, A.: Nanosatellite experiments to enable future space-based QKD missions, EPJ Quantum Technology, 3(1) (2016), pp. 12
18) Cheng, C., Chandrasekara, R., Tan, Y. C. and Ling, A.: Spacequalified nanosatellite electronics platform for photon pair experiments. Journal of Lightwave Technology, 33(23) (2015), pp. 4799 4804.

19) Durak, K., Villar, A., Septriani, B., Chandrasekara, R., Tang, Z., Bedington, R. and Ling, A.: The next iteration of the small photon entangling quantum system (SPEQS-2.0), SPIE OPTO,Proceedings of the International Society for Optics and Photonics, 2016.

20) Tan, Y. C., Chandrasekara, R., Cheng, C. and Ling, A.: Radiation tolerance of opto-electronic components proposed for space-based quantum key distribution, Journal of Modern Optics, 62(20) (2015), pp. 1709-1712.

21) Mukherjee, B., Wu, X., Maczka, T., Kwan, T., Huang, Y. and Mares, V.: Near space radiation dosimetry in Australian outback using a balloon borne energy compensated PIN diode detector, Radiation Measurements., 94 (2016), pp. 65-72.

22) Gomspace Nanocam C1U Datasheet, https://gomspace.com/UserFiles/Subsystems/datasheet/gs-dsnanocam-c1u-14.pdf (accessed March 23, 2017)

23) SpaceX - Dragon, http://www.spacex.com/dragon (accessed March $23,2017)$

24) Orbital ATK - Commercial Resupply Services, https://www.orbitalatk.com/space-systems/human-space-advancedsystems/commercial-resupply-services/ (accessed March 23, 2017)

25) H-II Transfer Vehicle KOUNOTORI (HTV), http://global.jaxa.jp/projects/rockets/htv/ (accessed March 23, 2017)

26) NanoRacks Smallsat Deployer, http://nanoracks.com/products/smallsatdeployment/ (accessed March 23, 2017)

27) Japanese Experimental Module - Small Satellite Orbital Deployer, http://iss.jaxa.jp/en/kiboexp/jssod/ (accessed March 23, 2017)

28) NanoRacks completes historic above space station Cygnus CubeSat Deployment, http://nanoracks.com/historic-above-space-stationcygnus-cubesat-deployment/ (accessed March 23, 2017)

29) Azur Space $30 \%$ triple junction GaAs solar cell assembly Datasheet, http://www.azurspace.com/images/products/0003401-0101_DB_3G30A.pdf (accessed March 23, 2017)

30) Gomspace - NanoPower Battery datasheet. https://gomspace.com/UserFiles/Subsystems/datasheet/gs-dsnanopower-batteries-10.pdf (accessed March 23, 2017)

31) Kwan, T., Wu, X., Kim, E., Bedington, R., Bai, X., and Ling, A.: Design and implementation of a high powered electrical power subsystem for AMMEQ-1, a 3U cubesat, Proceedings of 31st International Symposium on Space Technology and Science, Matsuyama, Japan, ISTS, 2017. 